\title{
Assessment of Stress Level of Young Undergraduates Before and After a Degree Examination Using Heart Rate Variability Analysis
}

\author{
A. Obi, E. O. Nwobodo, U. Dimkpa, S. O. Maduka, and E. Fintan
}

\section{ABSTRACT}

This study aimed at assessing the value of heart rate variability (HRV) as a stress indicator before and after a final re-sit exam among healthy sixth grade medical students. Fifty participants were recruited for the study (test group, $n=30$; control group, $n=20$ ). Each participant was examined for 5 minutes pre and post exam periods using the Heartmath proprietary protocol. EmWave equipment was used to detect, record and analyze the HR and to plot out the variability in discrete percentages for low, medium and high coherences. Results indicated that mean percentage coherence score was significantly higher in the test group $(p<0.05)$ at low cardiac coherence domain, but lower $(p<0.05)$ at the high coherence domain, compared with the control. Coherence score was significantly higher $(p<$ 0.05 ) after the exam indicating release from stress, as compared to before the examination when stress was observable among the exam candidates. There were no significant gender differences observed in cardiac coherence scores before and after examination. Our findings indicate that HRV is a reliable indicator of real-time exam stress and supports future clinical use of HRV as a non-invasive and simple stress test.

Keywords:Cardiac coherence, heart rate variability, pre and postexaminations, stress.

\section{INTRODUCTION}

Stress can be defined as a physical response or condition usually characterized by the symptoms of mental and physical tension or strain, resulting from a reaction to a situation in which an individual feels pressured or threatened or sometimes both [1]. Studies have indicated that examination situations and examination periods are usually stressful especially for medical students, because of the perceived stressful nature of medical education. The exam induced stress is reported to be associated with increased level of anxiety, depressiveness and negative emotional states which students experience at such times [2]-[5]. These
Submitted : August 9,2021

Published. : September 14,2021

ISSN: 2593-8339

DOI: $10.24018 /$ ejmed.2021.3.5.1008

A. Obi

Department of Human Physiology, Faculty of Basic Medical Sciences, Nnamdi Azikiwe University Awka, Nnewi Campus, Nigeria.

(e-mail: obiaugustus16@yahoo.com) E. O. Nwobodo

Department of Human Physiology, Faculty of Basic Medical Sciences, Nnamdi Azikiwe University Awka, Nnewi Campus, Nigeria.

(e-mail: eo.nwobodo@unizik.edu.ng) U. Dimkpa*

Department of Human Physiology, Faculty of Basic Medical Sciences, Nnamdi Azikiwe University Awka, Nnewi Campus, Nigeria.

(e-mail: u.dimkpa@unizik.edu.ng) S. O. Maduka

Department of Human Physiology, Faculty of Basic Medical Sciences, Nnamdi Azikiwe University Awka,

Nnewi Campus, Nigeria.

(e-mail: so.maduka@unizik.edu.ng)

E. Fintan

Neurology Unit, College of Medicine,

Enugu State University of Technology

Parklane GRA Enugu, Nigeria.

(e-mail:

fourcornersmedicals@gmail.com)

*Corresponding Author negative conditions are often triggered by fear of failure, heavy workload, lack of time to do revision, having difficulty understanding course content, anxiety over teacher's evaluation of performance, unpreparedness, and high expectations from family members, friends and teachers, as well as previous unfavorable examination experiences. It is normal to feel some degree of stress before exams; in fact, a small level of stress and anxiety can actually help a student perform well. However, excessive stress during exams will reduce effectiveness, reduce self esteem and hamper students' performance and achievement [6], and can lead to physical and mental health issues [7]. The physical, psychological, and behavioral abnormalities associated with 
chronic stress is reported to be due to hyperactivation of the sympathetic nervous system [8].

The search for stress biomarkers has remained a challenging task for clinicians and researchers. Presently, there is no universally recognized standard of evaluation for stress. This is due to several obstacles, which include lack of a comprehensive structure or set of rules for the investigation of how organisms adapt to and function in constantly changing environments and the lack of general agreement on the definition of stress [8], [9]. However, a considerable number of studies have used existing stress measurement methods such as psychological measures of stress (e.g., perceived stress scale, stress questionnaires), biochemical markers (e.g., cortisol, amylase) and physiological measures (e.g., heart rate (HR), blood pressure (BP) and heart rate variability) to measure examination stress [8]. Most of these investigations have shown that students' stress increases as their exams approach. For example, an increase in bad mood, bodily discomfort, restlessness, lack of energy, somatic strain was observed among students during pre-exam period by Huwe et al. [10]. Increase in immune-suppressive effects and cortisol levels have also been reported in previous studies that used adrenocortical, cardiovascular and immune system reactions, and they were found to be associated with examination stress [11]-[14]. Another study [15] also found higher diastolic BP and HR among medical students during a medical licensing exam. Although some of these stress measures proved to be good indicators of overall stress, they do not clearly reflect changes in mental component of the load, which is one of the major components of exam stress. However, stress parameters based on HRV are reported to be better and more sensitive to changes in mental load compared with other measures of stress [16]. Apart from being good indicators of stress during an exam, measures of stress involving cardiac activity parameters have also been shown to differentiate between the contributions of emotional and mental stress components to overall of exam stress [17].

Heart rate variability is a normal physiological phenomenon of the constant variation in the time interval in milliseconds, between successive heart beats, which reflects the activities of the sympathetic and parasympathetic components of the autonomic nervous system [18] [19]. Reliable HRV analysis therefore requires an accurate measurement of each heart beat and the time between beats and should also reflect the heart-brain interaction and changes in autonomic nervous system dynamics, which are particularly associated with stress and changes in emotional state of an individual [8]. An optimal level of HRV within an organism reflects healthy function and an inherent selfregulatory capacity, adaptability, and resilience [20]. Thus, HRV parameters will increase with decrease in mental load in tasks of various complexities [21]-[23]. Similar results were obtained in real working situations in offices, on pilots during flights, etc. [24], [25].

Several HRV biofeedback based technologies have been developed in order to assist people in controlled stress studies. Such technologies also allow users to use HRV as a measure of physiological coherence. Freeze-Framer, from the institute of Heartmath proposed heart rhythm coherence biofeedback based on HRV time frequency analysis. The emWave 2 technology of the HeartMath system selected for the present study basically involves heart focused breathing and feeling [26]. The emWave 2 is an advanced heart rate monitor, able to measure subtle changes in heart rhythms. It operates based on the cardiac coherence principle and demonstrates that mental and emotional states affect autonomic nervous system activity, HRV waveforms as well as the coupling between the heart and respiration. Thus, states of agitation, frustration, anger, anxiety and fear tend to cause disorder in HRV waveforms [27]. Coherence can be defined as a state of synchronization between the heart, brain and autonomic nervous system which has been proven to have numerous mental, emotional and physical benefits [28].

To the best of our knowledge no previous studies have assessed the level of stress before and after final re-sit examination among Nigerian medical students using the HRV analysis. Furthermore, despite the assumption that HRV is a reliable index of stress by a considerable number of authors, only few studies have confirmed whether it is a good indicator of stress before and after examinations. To support its future use as a noninvasive and simple diagnostic test, the value of HRV as a stress indicator must be assessed during pre-exam conditions to help in the management of stress especially among students preparing for exams.

In this study, we used the cardiac coherence technique, which is based on HRV time frequency analysis, to measure the level of stress among young Nigerian undergraduate medical students, before and after a degree examination. It is believed that the present study will provide a rationale for selecting HRV as a reliable indicator of mental stress and also provide health researchers across disciplines with a useful update on best practices for measuring mental stress.

\section{Methodology}

\section{A. Participants}

A total of 30 final year medical students (19 males, 11 females) aged between $24-33$ years $(27.10 \pm 0.70$ years $)$ and 20 age-matched controls ( 8 males, 12 females; mean age, $27.53 \pm 0.52$ years) voluntarily participated in the study. The test subjects included students who were preparing for a resit of their final MBBS degree examination. The control subjects were medical students who had already passed their MBBS exam and were not involved in any degree exam. The participants filled in a questionnaire regarding their individual health conditions, to ascertain if they had any chronic illness or if they were on any medication. Participants were also instructed to abstain from smoking, alcohol, caffeine or caffeinated drinks and other psycho-stimulants at least 24 hours before the commencement of each phase of the study. Exclusion criteria included history of specific health challenges such as cardiovascular diseases, severe tachycardia, and other disorders such as diabetes and hypertension. Other exclusion criteria included smoking history, alcoholic disease, obesity and taking medications that affect cardiovascular function. Before the commencement of the study, subjects were informed (written and oral) of the experimental procedures and their consents were obtained before participation. The Experiments and Ethics Committee of the College of Health Sciences, Nnamdi Azikiwe University, Nigeria approved the study. The guidelines and 
recommendations for studies involving humans were strictly adhered to.

\section{B. Study Procedure}

The participants were assessed for stress, anxiety and their psychophysiological coherence and HRV scores were recorded using the emWavetechnology of the HeartMathsystem (Model No: 2-01 5-8V, DC: 1.0A max). The study was divided into two phases for the test group. Phase I was executed at the point of entrance to the examination hall i.e. few minutes before the examination. Phase II was done within the first week after examination. The control group was also tested at the same period as the test group. For the purpose of this study, final year (600 Level) re-sit exam was chosen. This is because of its importance to the professional future of the students. A medical student who has to re-sit an exam especially in his or her final year is most likely to go through anxious moments because he/she must pass this exam before graduation from medical school and induction into the medical profession.

The participants were asked to sit comfortably in a quiet room of $28^{\circ} \mathrm{C}$ with forearm placed in mid or semi prone position and the wrist slightly flexed (for pulse regularity assessment at the radial pulse point). The participants while sitting comfortably in an unopposed setting with the head unsupported and feet on the ground, had the soft clip probe (ear sensor) of the Em-wave component applied to any of their earlobes from which the pulse is more active (for coherence assessment).

The soft clip ear lobe probe applied as above detects the heart rate, the EmWave unit in turn records and analyzes the HR and plots out the variability on the attached computer screen via the Emwave software. Furthermore, the software using a Heartmath proprietary protocol simultaneously generates and displays the cardiac coherence in discrete percentages for low, medium and high frequency band of HR oscillations. A total of 5 minutes was recorded per candidate in each phase of the study. Acquired data for each study participant from the above procedure was recorded manually in the log book of the study and the candidate was discharged for that phase.

\section{Statistical Analysis}

Data were expressed as Mean \pm Standard Error of Mean (SEM). Student's t-test was used to compare the mean coherence score between the two groups and phases. Test of statistical significance was set at $\mathrm{p} \leq 0.05$. All data analysis was performed using Statistical Package for Social Sciences (SPSS) version 25.0 (IBM-SPSS Statistics, 2017 Model, New York).

\section{RESULTS}

The mean pre-test scores of cardiac coherence for the control and test groups before the examination are presented and compared in Table I. Independent sample t-test indicated that at low cardiac coherence domain, the mean percentage score was significantly higher $(\mathrm{p}<0.05)$ in the test group $(51.37 \pm 2.61 \%)$ compared with the control $(40.30 \pm 2.75 \%)$. In the high cardiac coherence domain, the control group indicated significantly $(\mathrm{p}<0.05)$ greater percentage score compared with the test group (32.70 \pm 2.67 vs. $27.70 \pm 1.36$ ). On the other hand, there was no significant difference in mean percentage score between the two groups in the medium frequency coherence domain.

Table II shows the mean cardiac coherence scores in both control and test groups during the second phase (i.e. postexam period) of the study. Data showed no significant differences between the control and test group in all the cardiac coherence frequency domains (low, medium and high).

Table III shows the mean pre and post-test scores of cardiac coherence in the control group. Comparative analysis indicated lack of significant differences in the mean coherence scores between the pre and post tests at all frequency domains (low, medium and high).

The comparative analysis between the mean pre and posttest scores of cardiac coherence in the test group is expressed in Table IV. Result indicated significantly higher $(\mathrm{p}<0.01)$ percentage coherence score in pre-test $(51.37 \pm 2.61)$ compared with the post-test data $(35.73 \pm 2.74)$ in the low frequency domain. In the high frequency domain, significantly higher $(\mathrm{p}<0.01)$ mean cardiac coherence score was observed during the post-test $(34.40 \pm 3.42)$ compared with the pre-test period (21.27 \pm 2.12$)$. In contrast, there was no significant difference in percentage coherence score between the two test phases in the medium frequency domain.

Table $\mathrm{V}$ shows the mean percentage coherence scores before and after exams in both males and females of the test group. Independent sample t-test indicated that there were no significant gender differences in cardiac coherence scores of the test group before and after the examination at all frequency domains.

TABLE I: The MEAN PRE-TeSt SCORES OF CARDIAC COHERENCE FOR THE CONTROL AND TEST GROUPS BEFORE THE EXAMINATION (PHASE 1)

\begin{tabular}{ccccc}
\hline \hline Cardiac & & & & \\
Coherence & Control & Test Group & $\mathrm{t}-$ & $\mathrm{P}-$ \\
Frequency & $\mathrm{N}=20$ & $\mathrm{~N}=30$ & Statistics & Value \\
Domain & & & & \\
\hline \hline Low (\%) & $40.30 \pm 2.75$ & $51.37 \pm 2.61$ & -2.92 & $0.006^{*}$ \\
Medium (\%) & $32.70 \pm 2.67$ & $27.70 \pm 1.36$ & 0.55 & 0.585 \\
High (\%) & $38.15 \pm 2.69$ & $21.27 \pm 2.12$ & 2.67 & $0.012^{*}$ \\
\hline \hline *Significant difference at $\mathrm{p}<0.05$ & & &
\end{tabular}

TABLE II: THE MEAN POST-TEST SCORES OF CARDIAC COHERENCE FOR THE CONTROL AND TEST GROUPS AFTER THE EXAMINATION (PHASE II) Cardiac

\begin{tabular}{ccccc} 
Coherence & Control & Test Group & $\mathrm{t}-$ & $\mathrm{P}-$ \\
Frequency & $\mathrm{N}=20$ & $\mathrm{~N}=30$ & Statistics & Value \\
Domain & & & & \\
\hline Low (\%) & $36.05 \pm 2.66$ & $35.73 \pm 2.74$ & 0.08 & 0.934 \\
Medium (\%) & $32.10 \pm 2.66$ & $29.87 \pm 2.61$ & 0.59 & 0.552 \\
High (\%) & $31.85 \pm 3.58$ & $34.40 \pm 3.42$ & -0.51 & 0.609 \\
\hline
\end{tabular}

TABLE III: THE MEAN PRE AND POST-TEST SCORES OF CARDIAC COHERENCE IN THE CONTROL GROUP

\begin{tabular}{ccccc}
\hline \multicolumn{5}{c}{ COHERENCE IN THE CONTROL GROUP } \\
\hline Heart Rate & $\begin{array}{c}\text { Pre-Test } \\
\text { Coherence } \\
\text { Domain }\end{array}$ & $\begin{array}{c}\text { Post-Test } \\
\mathrm{N}=30\end{array}$ & $\begin{array}{c}\mathrm{t}- \\
\text { Statistics }\end{array}$ & $\begin{array}{c}\mathrm{P}- \\
\text { Value }\end{array}$ \\
\hline \hline Low (\%) & $40.30 \pm 2.75$ & $36.05 \pm 2.66$ & 1.11 & 0.27 \\
Medium (\%) & $32.70 \pm 2.67$ & $32.10 \pm 2.66$ & 0.95 & 0.35 \\
High (\%) & $38.15 \pm 2.69$ & $31.85 \pm 3.58$ & -1.94 & 0.06 \\
\hline \hline
\end{tabular}


TABLE IV: THE MEAN PRE AND POST-TeST SCORES OF CARDIAC COHERENCE IN THE TEST GROUP

\begin{tabular}{ccccc}
\hline \hline Cardiac Coherence & $\begin{array}{c}\text { Pre-Test } \\
\mathrm{N}=30\end{array}$ & $\begin{array}{c}\text { Post-Test } \\
\mathrm{N}=30\end{array}$ & $\begin{array}{c}\mathrm{t}- \\
\text { Statistics }\end{array}$ & $\begin{array}{c}\mathrm{P}- \\
\text { Value }\end{array}$ \\
\hline \hline Low (\%) & $51.37 \pm 2.61$ & $35.73 \pm 2.74$ & 4.13 & $0.001^{*}$ \\
Medium (\%) & $27.70 \pm 1.36$ & $29.87 \pm 2.61$ & -0.73 & 0.460 \\
High (\%) & $21.27 \pm 2.12$ & $34.40 \pm 3.42$ & -3.26 & $0.004^{*}$ \\
\hline \hline
\end{tabular}

*Significant difference at $\mathrm{p}<0.05$

TABLE V:. MEAn Percentage CoHerence SCORES Before AND AFTER EXAMS COMPARED BETWEEN MALES AND FEMALES OF THE TEST GROUP

\begin{tabular}{|c|c|c|c|c|c|}
\hline $\begin{array}{c}\text { Test } \\
\text { Phases }\end{array}$ & $\begin{array}{c}\text { Cardiac } \\
\text { Coherence } \\
\text { Frequency } \\
\text { Domain }\end{array}$ & $\begin{array}{l}\text { Males } \\
\mathrm{N}=19\end{array}$ & $\begin{array}{c}\text { Females } \\
\mathrm{N}=11\end{array}$ & $\begin{array}{c}\mathrm{t}- \\
\text { Statistics }\end{array}$ & $\begin{array}{c}\mathrm{P}- \\
\text { Value }\end{array}$ \\
\hline $\begin{array}{l}\text { Pre- } \\
\text { Exam }\end{array}$ & Low (\%) & $\begin{array}{l}51.52 \\
\pm 2.76\end{array}$ & $\begin{array}{c}51.09 \pm \\
5.49\end{array}$ & 0.08 & 0.938 \\
\hline \multirow{5}{*}{$\begin{array}{l}\text { Post- } \\
\text { Exam }\end{array}$} & Medium (\%) & $\begin{array}{l}28.15 \\
\pm 1.83\end{array}$ & $\begin{array}{c}26.90 \pm \\
1.99\end{array}$ & 0.43 & 0.665 \\
\hline & High (\%) & $\begin{array}{l}20.31 \\
\pm 1.83\end{array}$ & $\begin{array}{c}22.90 \pm \\
4.97\end{array}$ & -0.58 & 0.565 \\
\hline & Low $(\%)$ & $\begin{array}{l}36.26 \\
\pm 3.13\end{array}$ & $\begin{array}{c}34.81 \pm \\
5.34\end{array}$ & 0.25 & 0.804 \\
\hline & Medium (\%) & $\begin{array}{l}30.52 \\
\pm 3.35\end{array}$ & $\begin{array}{c}28.72 \pm \\
4.29\end{array}$ & 0.33 & 0.746 \\
\hline & High (\%) & $\begin{array}{l}33.21 \\
\pm 4.03\end{array}$ & $\begin{array}{c}36.45 \pm \\
6.42\end{array}$ & -0.45 & 0.656 \\
\hline
\end{tabular}

\section{DiscUSSION}

A considerable number of studies have associated vagally mediated HRV to self-regulatory capacity [29], emotion regulation [30], and the feeling of coherence of an individual [31]. The term cardiac coherence or physiological coherence is used to describe the measurement of the orderliness and stability in the synchronous and harmonious functioning of the regulatory systems of the body at any given time [28]. The coherence of the cardiac rhythm is reflected in the HRV power spectrum which comprises three frequency domains of low frequency $(0.01-0.05 \mathrm{~Hz})$, mid-frequency $(0.05-$ $0.15 \mathrm{~Hz})$ and high frequency $(0.15-0.50 \mathrm{~Hz})$ bands [32]. The low frequency signifies a time of cardiac incoherence (low cardiac coherence), when there is no wave-like activity in the HRV wave. It is predominantly controlled by the sympathetic nervous system and a high value is indicative of low HRV, increased sympathetic outflow and increased level of stress. A lower value is reflective of a more relaxed and restful condition. The medium coherence state signifies that there is some wavelike activity and it reflects baroreceptor reflex sensitivity and responsible for beat to beat blood pressure control [33]. It can be influenced by either sympathetic or parasympathetic activity or a mixture of both, but primarily from parasympathetic activity [33]. The HF domain represents high cardiac coherence state representing a higher percentage of time when there is wave-like activity. It is considered as an index of parasympathetic activity and a high value is indicative of increased $\mathrm{HRV}$, increased vagal outflow and decreased level of stress or state of relaxation [34], [35]. On the other hand, lower HF power is correlated with decreased HRV, decreased parasympathetic activity, and increased level of stress, panic, anxiety, or worry [36].

A previous data has shown that some soldiers diagnosed with post-traumatic stress disorder (PTSD) presented with both decreased coherence ratios and HRV scores than soldiers without PTSD [37].

The present study indicated that before the exam, percentage cardiac coherence score of the low frequency domain was significantly higher in the test group compared with the control. In contrast, the percentage coherence score was significantly lower in the test group compared with the control in the HF domain. These findings suggest that with a higher sympathetic activity of the heart as shown by the higher LF coherence score and a lower parasympathetic activity, reflected by a lower HF coherence score, the test group who were about taking a re-sit exam indicated a lower HRV in each case, hence a higher level of mental stress compared with the more relaxed control who were not taking any exams. This finding was expected and in agreement with previous studies, which have indicated that during examinations, students tend to have lower HRV compared to non-examination periods with an attendant decrease in parasympathetic activity of the heart in the HF domain and an increase in sympathetic activity as shown by an increase in the LF domain [38], [39]. Other studies have also reported a decline in HRV scores in the event of an increased mental load in various forms of tasks [21], [23]. The lack of significant differences in the MF cardiac coherence score may suggest no difference in baroreflex sensitivity to mental stress. A previous study has also reported no significant change in systolic blood pressure in medical students undergoing a medical licensing examination during exam compared to a non-exam period [15]. Interestingly, our result indicated that at post-examination period, the test group data recovered back to match with those of the control at both LH and HF domains. This suggests that during the restful or less stressful post-exercise period, the previously stressed students may have experienced increased parasympathetic activity leading to an increased HRV, which is associated with less energy mobilization and greater range of possible adaptive responses [40], [41]. The tilting of cardiac coherence toward stability and increased HRV under less stress may be as a result of decoupling of multimodal integrated networks [42].

The test students in our study presented a higher mean coherence score before the exam compared to after the exam in the LF coherence domain, whereas they presented lower mean coherence score before exam compared with post-exam in the HF domain. These findings suggest that in both cardiac coherence domains, the pre-exam period indicated a condition of lower HRV, higher sympathetic outflow and higher level of mental stress, anxiety and worry compared with the post exam condition, which is considered as a relaxation period and characterized by a restoration of the parasympathetic dominance and HRV augmentation. Our finding is in tandem with previous studies which indicated lower HRV before exam compared with after exam [43] or non-exam period [38], [44]. In contrast, another study indicated no significant difference in HRV when comparing an examination day to a normal academic day [39]. It is noteworthy that among the control, there were no changes in cardiac coherence score in all the frequency domains. This suggests that the HRV is at its optimal state among the control group and the students were in relaxed state of mind in both phases of our study. Our finding also agrees with previous 
reports which showed that parasympathetic nervous system is dominant during restful or less stressful events [45], [46].

Furthermore, our study indicated that among the test group, the male students presented the same cardiac coherence scores as the females at all levels. This suggests that the HRV and the level of mental stress did not differ between males and females. This result is in contrast with previous studies, which have shown that in exam stress conditions, men possess higher HRV compared with women [47], [48].

The limitations of the present study include the age range of participants recruited for the study (24-33 years), which may restrain the extension of its application to participants of a wider age population. Other limitations include the recruitment of unequal number of participants in both control and test groups as well as in both genders and the moderate sample size of the study (due to the unwillingness of majority of the medical students' population to participate). These may have impacted on the strength of significance in comparative analysis between groups.

\section{CONCLUSION}

Stress levels among the medical students taking their final re-sit exam appeared to be higher before the exam as indicated by a decrease in cardiac coherence, when compared with the relaxed, less stressed control. During the mental stress stimulation (pre-exam period), components of the sympathetic nervous system increased as shown by an increase in LF score and with a decrease in the values of parasympathetic activity as shown by a decrease in HF scores among the test group. These changes were reversed after the stressor (exam) was over, when factors reflecting sympathetic activity decreased and a predominance of parasympathetic activity was restored resulting in lack of significant differences in cardiac coherence scores between the control and test group. The same parameters also showed a decline in stress level (higher HRV) at all domains among the test group, during the post-exam situations compared with preexam period. This study therefore validates the previous claims that HRV is a reliable indicator of stress and supports future clinical use of HRV as a non-invasive and simple stress test. Overall, the present findings indicated that final year resit examination time at the medical school is considered a stressful period for the students. It can also be concluded that both the male and female students were prone to the same level of mental stress during their final re-sit degree exam. This present study therefore calls for a need to constantly evaluate the level of mental stress among medical students before examinations in order to provide an effective stress management and counseling program required for their optimum performance during exams. This will help avoid adverse health consequences of stress among undergraduate medical students in our universities.

\section{ACKNOWLEDGMENT}

The authors wish to thank the following for their support, encouragement, cooperation and commitment throughout the duration of the study; the head and staff of Region Hospital, Enugu; the head and staff of Human Physiology Department,
Nnamdi Azikiwe University, Nnewi Campus; the final year medical students from Enugu State University of Science and Technology, who participated in the study; and Prof. Jude Egwurugwu for his scholarly advise.

\section{REFERENCES}

[1] S. I. Haider, "Effect of Stress on Academic Performance of Undergraduate Medical Students," Journal of Community Medicine \& Health Education, Vol. 7, No. 06, DOI: 10.4172/2161-0711.1000566, 2017.

[2] G. Spangler, R. Pekrun, K. Kramer, and H. Hofmann, "Students' Emotions, Physiological Reactions, and Coping In Academic Exams", Anxiety. Stress. Coping, Vol. 15, No. 4, pp. 413-6, 2002.

[3] N. G. Vivian, D. Koh, and S.E. Chia, "Examination Stress, Salivary Cortisol and Academic Performance". Psychol. Rep., Vol. 93, pp. 1133-7, 2003.

[4] M. Oaten, and K. Cheng, "Academic Examination Stress Impairs Self Control,” J. Soc. Clin. Psychol., Vol. 24, pp. 254-279, 2005.

[5] D. Marazziti, F. Ambrogi, M. Abelli, E. D. Nasso, M. Catena, G. Massimetti, et al, "Lymphocyte Subsets, Cardiovascular Measures and Anxiety State Before and after a Professional Examination," Stress., Vol. 10, pp. 93-9, 2007.

[6] K. Rafidah, M. A. Azizah, M. Mohd, M. D. Norzaidi, M. Siong, and C. Chong, "Stress and academic performance: Empirical evidence from university students," Acad Educ Leadersh J., Vol. 13, pp. 37-51, 2009.

[7] S. Senarath, "Examination Stress, Stress Management Strategies, and Counseling Needs of College Level Students in Sri Lanka," Journal of Psychology and Behavioral Science, Vol. 8, No. 2, pp. 27-34, 2020.

[8] H. Kim, E. Cheon, D. Bai, Y.H. Lee., and B. Koo, "Stress and Heart Rate Variability: A Meta-Analysis and Review of the Literature," Psychiatry Investig, Vol. 15, No. (3), pp. 235-245, 2018.

[9] J. F. Thayer, F. Ahs, M. Fredrikson, J.J. Sollers, and T. D. Wager, "A meta-analysis of heart rate variability and neuroimaging studies: implications for heart rate variability as a marker of stress and health" NeurosciBiobehav Rev, Vol. 36, pp. 747-756, 2012.

[10] S. Huwe, J. Henning, and P. Netter, "Biological, Emotional, Behavioral and Coping Reactions to Examination Stress in High and Low State Anxious Subjects," Anxiety. Stress. Coping, 11, 47-65, 1998.

[11] G. Spangler, "Psychological and Physiological Responses during an Exam and Their Relation to Personality Characteristics," Psychoneuroendocrinology, Vol. 22, pp. 423-41, 1997.

[12] G. D. Marsall, S. K. Agarwal, C. Lloyd, and L. Cohen, E. M. Henninger, G. J. Morris, "Cytokine Dysregulation Associated with Exam Stress in Healthy Medical Students” Brain. Behav. Immun., Vol. 12, pp. 297-307, 1998.

[13] N. G. Vivian, D. Koh, and S.E. Chia, "Examination Stress, Salivary Cortisol and Academic Performance" Psychol. Rep., Vol. 93, pp. 11337, 2003.

[14] R. S. Lewis, and A. Nikolova, D.J. Chang, N. Y. Weekes, "Examination Stress and Components of Working Memory." Stress, Vol. 11, pp. 108-14, 2008.

[15] A. Zeller, D. Handschin, N. Gyr, B. Martina, and E. Battegay, "Blood Pressure and Heart Rate of Students Undergoing a Medical Licensing Examination," Blood. Press., Vol. 13, pp. 20-24, 2004.

[16] N. Šimic, and I. Manenica, "Exam Experience and Some Reactions to Exam Stress," Human Physiology, Vol. 38, pp. 67-72, 2012.

[17] N. Simic, "Evaluation of Exam Stress on the Basis Changes of Sinus Arrhythmia Parameters (in Croatian)," Acta Med. Croatica., Vol. 60, pp. 27-33, 2006.

[18] M. Malik, "Guidelines Heart rate variability," Eur. Heart J., Vol. 17, pp. 354-381, 1996.

[19] G. G. Berntson, J. T. Bigger, D. L. Eckberg, P. Grossman, P.G. Kaufmann, M. Malik, et al, "Heart rate variability: origins, methods, and interpretive caveats," Psychophysiology, Vol. 34, pp. 623-648, 1997.

[20] D. Nunan, G.R. Sandercock, and D.A. Brodie, "A quantitative systematic review of normal values for short-term heart rate variability in healthy adults," Pacing Clin Electrophysiol., Vol. 33, No.(11), pp. 1407-17, 2010.

[21] I. Manenica, and N. Krošnjar, "Effects of Mental Load and Paced Work on Parameters of Some Psychopysiological Variables (in Croatian)," RadoviFilozofskogfakulteta u Zadru, Vol. 29, pp. 95-107, 1990.

[22] R. W. Backs, J.K. Lenneman, and J. L. Sicard, "The Use of Autonomic Components to Improve Cardiovascular Assessment of Mental Workload in Flight Simulation," Int. J. Aviation Psychology, Vol. 9, pp. 33-47, 1999. 
[23] H. M. Reims, K. Sevre, and A. Hoieggen, "Blood Viscosity: Effects of Mental Stress and Relations to Autonomic Nervous System Function and Insulin Sensitivity," Blood. Press., Vol. 14, pp. 159-69, 2005.

[24] P. G. Jorna, "Heart Rate and Workload in Actual and Simulated," Flight. Ergonomics, Vol. 26, pp. 1043 - 54, 1993.

[25] A. H. Garde, and B. Laursen, A.H. Jorgensen, B. R. Jensen, "Effects of Mental and Physical Demands on Heart Rate Variability during Computer Work," Eur. J. Appl. Physiol., Vol. 87, pp. 456-61, 2002.

[26] S. Jasubhai, "Effect of Quick Coherence Technique on Psychophysiological Coherence, Heart Rate, Stress, Anxiety, Depression and Feeling State in Young Adults in India,"J Psychol Neurosci, Vol. 3, No. 1, pp. 1-9, 2021.

[27] R. McCraty, M. Atkinson, W.A. Tiller, G. Rein, and A.D. Watkins, "The effects of emotions on short-term power spectrum analysis of heart rate variability," American Journal of Cardiology, 76(14), 1089 1093, 1995.

[28] R. McCraty, and M.A. Zayas, "Cardiac coherence, self-regulation, autonomic stability and psychosocial well-being," Frontiers in Psychology, Vol. 5, No. 1090, 2014. DOI: 10.3389/ fpsyg.2014.01090.

[29] A. Reynard, R. Gevirtz, R. Berlow, M. Brown, and K. Boutelle, "Heart rate variability as a marker of self-regulation," Appl. Psychophysiol.Biofeedback, Vol. 36, pp. 209-215, 2011.

[30] F. Geisler, N. Vennewald, T. Kubiak, and H. Weber, "The impact of heart rate variability on subjective well-being is mediated by emotion regulation," Pers. Individ. Dif., Vol. 49, pp. 723-728, 2010.

[31] G. A. Miller, G.A., E. Galanter, E., and K.H. Pribram, Plans and the Structure of Behavior. New York: Henry Holt \& Co, 1960.

[32] R. E. Kleiger, P.K. Stein, M.S. Bosner, and J.N. Rottmann, "Time domain measurements of heart rate variability," AmbulElectrocardiol., Vol. 10, pp. 487-498, 1992.

[33] R. McCraty, M. Atkinson, D. Tomasino, and R.T. Bradley, The Coherent Heart Heart-Brain Interactions, Psychophysiological Coherence, and the Emergence of System-Wide Order HeartMath Research Center, Institute of HeartMath, Boulder Creek, CA, USA: 2006, pp. 6-22.

[34] G A. Reyes del Paso, W. Langewitz, L.J.M. Mulder, A. van Roon, and S. Duschek, "The utility of low frequency heart rate variability as an index of sympathetic cardiac tone: a review with emphasis on a reanalysis of previous studies," Psychophysiology, Vol. 50, 477-87, 2013.

[35] S. Laborde, and E. Mosley, J.F. Thayer, "Heart Rate Variability and Cardiac Vagal Tone in Psychophysiological Research Recommendations for Experiment Planning, Data Analysis, and Data Reporting," Front Psychol., Vol. 8, No. 213, 2017. https://doi.org/10.3389/fpsyg.2017.00213.

[36] F. Shaffer, and J.P. Ginsberg, "An Overview of Heart Rate Variability Metrics and Norms," Front Public Health, Vol. 5, No. 258, 2017.

[37] J. P. Ginsberg, M.E. Berry, and D.A. Powell, "Cardiac coherence and PTSD in combat veterans," Altern. Ther. Health Med, Vol. 16, pp. 5260, 2010.

[38] D. A. Dimitriev, A. D. Dimitriev, Y. D. Karpenko, and E. V. Saperova, "Influence of examination stress and psychoemotional characteristics on the blood pressure and heart rate regulation in female students," Hum. Physiol, Vol. 34, pp. 617-624, 2008.

[39] E. Tharion, S. Parthasarathy, and N. Neelakantan, "Short-term heart rate variability measures in students during examinations," Natl. Med. J. India, Vol. 22, pp. 63-66, 2009.

[40] F. E. Dewey, J. V. Freeman, G. Engel, R. Oviedo, N. Abrol, N. Ahmed, et al, "Novel predictor of prognosis from exercise stress testing: Heart rate variability response to the exercise treadmill test," Am. Heart J., Vol. 153, pp. 281-288, 2007.

[41] D. A. Dimitriev, E. V. Saperova, F. Z. Ross, and I. M. Sechenova, "Heart rate variability and blood pressure during mental stress" Ross FiziolZhIm I M Sechenova, Vol. 101, pp. 98-107, 2015

[42] C. Schubert, M. Lambertz, R. A. Nelesen, W. Bardwell, J. B. Choi, and J. E. Dimsdale, "Effects of stress on heart rate complexity - A comparison between short-term and chronic stress" Biol Psychol., Vol. 80(3), pp. 325-332, 2009.

[43] S. Hammoud, R. Karam, R. Mourad, I. Saad, and M. Kurdi, "Stress and Heart Rate Variability during University Final Examination among Lebanese Students,"Behav. Sci., Vol. 9, No. 3, 2019. doi:10.3390/bs9010003.

[44] V. N. Zaripov, and M. O. Barinova, "Changes in parameters of tachography and heart rate variability in students differing in the level of psychoemotional stress and type of temperament during an academic test week," Hum. Physiol., Vol. 34, pp. 454-460, 2008.

[45] A. U. Rajendra, J. K. Paul, N. Kannathal, C. M. Lim, and J. S. Suri, "Heart rate variability: a review," Med Biol EngComput, Vol. 44, pp. 1031-1051, 2006.
[46] S. Rotenberg, and J. J. McGrath, "Inter-relation between autonomic and HPA axis activity in children and adolescents," Biol Psychol, Vol. 117, pp. 16-25, 2016.

[47] M. Lutfi, and M. Y. Sukkar, "The Effect of Gender on Heart Rate Variability," Int. J. Health Sci., Vol. 5, pp. 146-154, 2011.

[48] R. P. Sloan, M. Huang, H. Mccreath, K. Liu, O. D. Williams, and T. Seeman, "Cardiac autonomic control and the effects of age, race and sex: The cardia study,” Auton. Neurosci. Vol. 139, pp. 78-85, 2008. 